\title{
Versuch einer mathematischen Analyse der „Normalkurve” von Krogh
}

\author{
Von Friedrich Krüger \\ Biologische Anstalt Helgoland \\ Zentrale Hamburg-Altona, Palmaille 9
}

Mit 10 Abbildungen und 9 Tabellen

\section{Summary}

The mathematical expression of biological temperature functions on the basis of VAN'T Horrs rule is unsatisfactory. This fact led Krogu (1914) to present a standard curve, which was meant to demonstrate the basic uniformity of temperature relationships of numerous biological processes. This standard curve has served as a reference point in many papers up to the present time. Simple comparison of curve shapes is difficult for carious reasons and does not allow exact statements. KrogH himself has not attempted to define the shape of his standard curve mathematically. A pertinent proposal by JøRGENS়EN (1916) remained unnoticed by most biologists, although it was suitable mathematically.

Last year I have proposed a modification of the ARRHenius formula for expressing biological processes mathematically, in which, instead of the absolute zero $\left(-273^{\circ} \mathrm{C}\right)$, a smaller value appeared as biological zero temperature:

$$
Y_{t}=\underset{m}{1 / t-z}
$$

where $Y_{t}=$ speed of the process at temperature $t ; m=$ maximum speed of the process at $\mathrm{t}=; \mathrm{z}=$ biological zero; $\mathrm{n}=\mathrm{a}$ very great number. The three parameters can be calculated from observed data. Only the determination of $z$ is somewhat more difficult.

The application of the new formula to several examples from literature led to very good results. In the present paper it is applied to the observed values on which Krogr based his standard curve. This procedure demonstrates that the new formula represents the values in many cases with a high degree of exactness. In addition, the new curve allows the representation of the numbers given by KROGH on the relation between temperature and respiration in Tenebrio-pupae, which could not be brought into coincidence with the standard curve.

A number of further examples have thus demonstrated that the new formula is suited for an exact representation of biological temperature functions. Single numbers - like the Q10 or $\mu$ values - cannot express biological temperature functions. The three parameters contained in the new formulare are the minimum requirements for a quantitative reproduction of a curve pattern.

The new formula has also been applied to express the relation between temperature and the speed of development of Aedes taeniorhynchus. In this case too it proves to be superior to the method of Jorgensen.

\section{Einleitung}

Der dänische Physiologe A. KroGH veröffentlichte 1914 eine Arbeit, in der er als Ergebnis von sehr sorgfältigen Versuchen über die Temperatur- 
abhängigkeit des Stoff wechsels einiger Tiere eine "Normalkurve“ entwarf, der sich seine Ergebnisse mehr oder minder gut anpaßten. Diese Normalkurve hat bis in die neueste Zeit hinein als Bezug für die Darstellung der Temperaturabhängigkeit des Stoffwechsels - aber auch der Entwicklungsgeschwindigkeit von Tieren - in einer Reihe von Untersuchungen gedient. KroGH kam zu dieser Lösung des Problems, weil er klar erkannte, daß die seit Van't Hofr (1896) übliche Wiedergabe der Temperaturabhängigkeit biologischer Vorgänge durch den $Q_{10}$-Wert, der die Reaktionsbeschleunigung bei einer Temperaturerhöhung um $10^{\circ}$ angibt, für eine Darstellung der Temperaturabhängigkeit biologischer Prozesse in der Mehrzahl der Fälle ungeeignet ist; denn selbst in dem engen Temperaturbereich, der für biologische Messungen zur Verfügung steht, kann von einer Konstanz des $Q_{10}$-Wertes keine Rede sein. Der $Q_{10}$-Wert bewegt sich zwar in vielen Fällen, entsprechend den Angaben von VAN'T Hoff, zwischen 2 und 3, also in relativ engen Grenzen, aber wir kennen viele Fälle, in denen er über diese Grenzen hinausgeht. Aber was wichtiger ist, $Q_{10}$ stellt keine die Temperaturabhängigkeit eindeutig charakterisierende Größe dar. Der $\mathrm{Q}_{10}$-Wert hängt, worauf BACH (1958) kürzlich mit Recht hinwies, von dem Temperaturintervall $a b$, aus dem er berechnet wird.

Die mathematische Grundlage der sog. Van't Horrschen Regel, die im Anschluß an KanITZ (1915) auch als RGT-Regel bezeichnet wird, bildet die von Berthelot (1862) für die Temperaturabhängigkeit chemischer Prozesse abgeleitete Formel:

$$
\mathrm{k}_{\mathrm{t}}=\mathrm{a} \cdot \mathrm{b}^{\mathrm{t}}
$$

Gemäß dieser Formel ist der Logarithmus der Reaktionsgeschwindigkeit $\left(k_{t}\right)$ proportional dem linearen Wert der Temperatur $(t)$. a und $b$ stellen in Gleichung (1) Konstanten dar. Die Prüfung auf das Zutreffen der RGT-Regel erfolgt am einfachsten durch Eintragung der Versuchswerte in ein semilogarithmisches Koordinatensystem, ein Verfahren, das bereits 1914 von PütTER angewandt wurde. Bei diesem Vorgehen müssen die Meßwerte als Funktion der Temperatur auf einer Geraden liegen, wenn sie der RGT-Regel folgen. Bei biologischen Vorgängen ist das nur ausnahmsweise der Fall. Fast immer erhält man mehr oder weniger stark gekrümmte Kurven, die anzeigen, daß die mathematischen Bedingungen der Formel von BerTHELot nicht erfüllt sind. Im allgemeinen verlaufen die so gezeichneten Kurven bei tieferen Temperaturen steiler als bei hohen Temperaturen, was zahlenmäßig seinen Ausdruck in der Abnahme des $Q_{10}$-Wertes bei steigender Temperatur findet.

Da die gleiche Erscheinung sehr häufig auch bei chemischen Reaktionen auftritt, schlug Arrhenius (1889) eine andere Formulierung für die Temperaturabhängigkeit der Reaktionsgeschwindigkeit vor, bei der im Gegensatz zu der Formel von Berthelot im Exponenten der reziproke Wert der absoluten Temperatur steht. Prinzipiell hat die Gleichung von Arrhenius die Gestalt:

$$
\mathrm{k}_{\mathrm{T}}=\alpha \cdot \beta-\frac{1}{\mathrm{~T}}
$$

$\mathrm{k}_{\mathrm{r}}$ stellt in der Gleichung die Reaktionsgeschwindigkeit bei der absoluten Temperatur $\mathrm{T}$ dar; die Konstante $\alpha$ ist die Reaktionsgeschwindigkeit für $\mathrm{T}=\infty$; $\beta$ ist eine zweite Konstante, die in der Formulierung von Arrhenius ihren Ausdruck in der Aktivierungsenergie A (von den Chemikern auch mit E, von den Biologen mit $\mu$ bezeichnet) findet.

Während sich in der Chemie die Formel von Arruenrus in vielen Fällen 
zur mathematischen Darstellung der Temperaturabhängigkeit von Reaktionen bewährt, hat sie in der Biologie nicht die auf sie gesetzten Erwartungen erfüllt.

Auch Gleichung (2) prüft man am einfachsten durch Darstellung der Versuchswerte im semilogarithmischen Koordinatensystem, nur daß man in diesem Fall die Logarithmen der Reaktionsgeschwindigkeit als Funktion des reziproken Wertes der absoluten Temperatur darstellt. Bei Stoff wechselversuchen setzt man als Ausdruck für die Reaktionsgeschwindigkeit den Stoffumsatz, bei Atmungsmessungen dementsprechend den Sauerstoffverbrauch ein.

Der enge Temperaturbereich, der für biologische Messungen in Frage kommt und sein großer Abstand von dem absoluten Nullpunkt machen verständlich, daß bei der graphischen Darstellung die reziproken Werte der absoluten Temperaturgrade nahezu äquidistant sind und sich daher die Kurven nach Gleichung (1) und (2) kaum voneinander unterscheiden. Aus diesem Grunde ließ sich auch bis heute bei biologischen Objekten nur in Ausnahmefällen eine eindeutige Entscheidung zugunsten der einen oder anderen Formulierung geben. Im allgemeinen ergab sich, daß biologische Temperaturfunktionen auch nicht näherungsweise durch Gleichung (1) oder (2) in einem weiteren Temperaturbereich darzustellen sind.

So wird verständlich, daß die Formel von Arrhensus, die keine nennenswerten Vorzüge vor dem $Q_{10}$-Wert besitzt, da auch die $\mu$-Werte nicht konstant sind, nicht allgemeinen Eingang in die Biologie gefunden hat und auch heute noch die Beschreibung der Temperaturabhängigkeit biologischer Prozesse durch die Angabe von $Q_{10}$-Werten erfolgt, ungeachtet der Tatsache, daß dieses Verfahren im Grunde genommen wenig Sinn hat.

Unter diesen Umständen war das Vorgehen KroGus (1914), den oft sehr ähnlichen Verlauf der Temperaturkurven biologischer Vorgänge durch eine Normalkurve darzustellen, rein objektiv betrachtet, richtiger als die Angabe der in keiner Weise eindeutig die Temperaturabhängigkeit charakterisierenden $Q_{10}$-Werte. KroGir hat seine Normalkurve auf Grund von Messungen an relativ wenigen Objekten entworfen: und zwar an einem Goldfisch, einigen Fröschen und Kröten sowie einem Hund. Eine möglichst weitgehende Deckung mit seiner Normalkurve wurde bei der unterschiedlichen Atmungsgröße der Objekte durch Multiplikation mit geeignet gewählten Faktoren erreicht.

Im ganzen genommen befriedigt die Darstellung der Temperaturfunktion durch die Normalkurven nicht sehr; nicht nur, daß sie in vielen Fällen nicht anwendbar ist - KROGH selbst bringt als Beispiel die Atmung von TenebrioPuppen -, sondern die Kurve ist auch mathematisch nicht eindeutig definiert und daher die Frage der Deckung der Versuchspunkte mit der Kurve weitgehend eine Ermessensfrage. Es wäre daher als ein wesentlicher Fortschritt zu betrachten, wenn es gelingt, die der KroGHschen Normalkurve zugrunde liegende mathematische Funktion zu erfassen. Der gleichartige Verlauf der Temperaturkurven systematisch recht verschiedener Versuchstiere legt den Verdacht nahe, daß ihnen eine gewisse Gesetzmäßigkeit zugrunde liege und daß es daher nicht aussichtslos sei, einen mathematischen Ausdruck dafür zu suchen. KroGH selbst hat sich anscheinend darum nicht bemüht. Auf einen entsprechenden Versuch von Jørgensen werde ich später zu sprechen kommen.

Vor das Problem gestellt, die Temperaturabhängigkeit der Atmung der Miesmuschel exakt darzustellen, die im Verlauf des Jahres — nach noch nicht veröffentlichten Versuchen - deutlich erkennbare Änderungen zeigt, ergab sich die zwingende Notwendigkeit, die jahreszeitlichen Variationen eindeutig wie- 
dergeben zu können. Die bislang in der Biologie übliche Darstellung durch ! oder $Q_{10}$-Werte hätte zu umständlichen Aufzählungen von solchen Werten geführt, die höchstens eine oberflächliche Beschreibung der Befunde ermöglichten. Im Prinzip lieferten diese Messungen Kurven, die der Kroghschen Normalkurve ähnlich waren.

Ich ging nun von dem Grundgedanken aus, daß die Formel von ArrheNius prinzipiell zwar der Abnahme der Reaktionsbeschleunigung bei steigender Temperatur, wie man sie bei biologischen Prozessen allgemein findet, gerecht wird, daß aber durch den großen Abstand der Meßtemperatur vom absoluten Nullpunkt die Krümmung der Kurve in dem in Frage kommenden Temperaturbereich zu schwach ist, um biologische Temperaturfunktionen wiederzugeben.

Die dem Exponenten der Arrhenrus-Formel zugrunde liegende Reihe:

$$
\frac{1}{0} ; 1 ; \frac{1}{2} ; \frac{1}{3} ; \frac{1}{4} ; \cdot . \cdot . \quad . \quad \frac{1}{\infty}
$$

stellt bei der graphischen Wiedergabe eine Kurve dar, die am Anfangsteil sehr steil abfällt und um so flacher verläuft, je größer der Nenner wird. Es bietet diese Reihe also sehr verschiedene Krümmungsgrade, und wenn man im $\mathrm{Ex}$ ponenten der Gleichung (2) nicht den absoluten Nullpunkt von $-273^{\circ}$ als Bezugstemperatur wählt, sondern eine höhere Temperatur, gelangt man in Kurvenbereiche, die eine stärkere Krümmung zeigen. Ein solches Vorgehen erscheint bei biologischen Prozessen berechtigt, da ihr Nullpunkt sicherlich bei höheren Temperaturen als dem absoluten Nullpunkt zu suchen ist.

In der. Tat zeigte sich, daß bei geeigneter Wahl der Nulltemperatur die Meßspunkte von Mytilus mit einer für biologische Meßwerte hinreichenden Genauigkeit im semilogarithmischen Koordinatensystem durch eine Gerade darzustellen waren. Gleichung (2) gab ich in Anpassung an die biologischen Bedürfnisse die Form:

$$
y_{t}=-m \frac{1}{n^{t-z}}
$$

In ihr stellt y den Stoffumsatz dar - in unserem speziellen Fall den Sauerstoffverbrauch - $m$ und $n$ sind Parameter, und zwar ist $m$ die maximale Leistungsfähigkeit des Systems, n ist eine sehr große Zahl, die daher als dekadischer Logarithmus angegeben wird, $t$ ist die Meßtemperatur und $z$ ist die Nullpunkttemperatur. Beide Temperaturwerte sind entsprechend ihrem Vorzeichen in die Gleichung einzusetzen. Die bislang nur negativ gefundenen Werte für $z$ sind also zur Meßtemperatur zu addieren.

Die jahreszeitlichen Änderungen in der Temperaturabhängigkeit der Atmung von Mytilus erfordern langfristige Versuchsserien und erlauben fürs erste nicht, sie den weiteren Ausführungen zugrunde zu legen. Aus diesem Grunde habe ich (1961) einige Beispiele aus der Literatur auf die Anwendbarkeit von Gleichung (3) geprüft. Hierbei zeigte sich, daß das vorgeschlagene Prinzip über den speziellen Fall hinausgehend allgemeinerer Anwendung fähig ist. Schon bei der ersten durchgeführten mathematischen Analyse der Versuchszahlen von Јов (1955) für die Atmung von Salvelinus fontinalis ergaben sich sehr interessante Einblicke in die sehr komplexe Natur biologischer Temperaturfunktionen, für die auf die genannte Arbeit verwiesen werden muß. 
Auswertung der Meßergebnisse von KroGH
nach Gleichung (3)

Der ähnliche Verlauf der bislang nach Gleichung (3) dargestellten Temperatur-Funktionen mit der Krogrsschen Normalkurve ließ vermuten, daß hiermit auch der mathematische Ausdruck für diese gegeben sei.

Die Auswertung der Versuchszahlen von Krogh trifft auf gewisse Schwierigkeiten. Erstens umfassen sie zum Teil nur enge Temperaturspannen und zweitens geben sie verschiedentlich für gleiche oder nahe beieinanderliegende Temperaturen sehr stark schwankende Werte. Die im Folgenden angegebenen Parameterwerte können infolgedessen nicht als vollkommen zuverlässig angesehen werden. Für eine exakte Analyse wären erneute Messungen durchzuführen. An dieser Stelle kann nur demonstriert werden, daß es grundsätzlich möglich ist, Stoffwechselkurven von dieser Art durch Gleichung (3) mathematisch wiederzugeben.

Die hierbei zu lösende Aufgabe ist, aus den Versuchswerten den Wert für z zu bestimmen. Verhältnismäßig einfach kann man diesen näherungsweise graphisch ermitteln, indem man $z$ variiert und prüft, bei welchem Wert von $z$ die Versuchspunkte im semilogarithmischen Koordinatensystem mit größter Näherung als Funktion von $1 / t-z$ durch eine Gerade darzustellen sind. In diesem Falle sind die Bedingungen der Gleichung (3) erfüllt. Aus dem gefundenen $z$-Wert lassen sich die beiden anderen Parameter mit Hilfe der Meßwerte leicht berechnen.

Die rechnerische Bestimmung von $z$ aus den Versuchswerten ist durch seine Lage im Nenner des Exponenten recht umständlich. Daher empfiehlt sich die Benutzung einer elektronischen Rechenanlage. Für den genauer analysierten Fall der Temperaturabhängigkeit der Atmung von Salvelinus (Јов 1955) hatte Herr Dr. Nicolovius vom Institut für angewandte Mathematik an der Universität Hamburg entgegenkommenderweise diese Aufgabe übernommen und größenordnungsmäßig die graphisch ermittelten Werte bestätigen können. Grundsätzlich dürfte die rechnerische Bestimmung von $\mathrm{z}$ vorzuziehen sein, da sie die Gefahr einer subjektiven Beurteilung ausschließt, sie setzt aber ein sehr zuverlässiges und geeignetes Zahlenmaterial voraus. Für die von Кrogh gemessenen Werte trifft das nicht zu.

Herr Dr. Nicolovius war so freundlich, sich der großen Mühe zu unterziehen, auch aus den von Квоян gegebenen Zahlen die Parameter der Gleichung (3) zu berechnen, wobei sich erwies, daß die Güte der Meßwerte von größtem Einfluß auf die Berechnung ist. Während bei guten Zahlenreihen das Ausschalten einzelner Meßwerte praktisch ohne Einfluß auf das Ergebnis war, ergab es in anderen Fällen grundsätzlich andere Werte.

Die Verarbeitung der Versuchszahlen von KroGH führte noch zu einer wesentlichen methodischen Erkenntnis. Bei der Berechnung der Parameter aus den Daten von Joв wurde $z$ so gewählt, daß das Quadrat der linearen $A b$ weichungen der Versuchswerte von den berechneten Werten zum Minimum wurde.

$$
i \stackrel{k}{=}\left[y_{i}-e^{b-} \stackrel{a}{t-z}\right]^{2}=\operatorname{Min} .
$$

Bei den Versuchszahlen von Krogh führte dieser Weg zu z-Werten, die sich bei der graphischen Kontrolle als unbrauchbar erwiesen. Mit der graphi- 
schen Darstellung vereinbarte $z$-Werte ergaben sich dagegen, wenn die Differenz zwischen den Logarithmen der Versuchswerte und der berechneten Werte der Minimum-Forderung entsprach.

$$
\stackrel{i}{=}\left[1 \ln y_{i}-\left(b-\frac{a}{t_{i}-z}\right)\right]^{2}=\text { Min. }
$$

Die Ursache hierfür dürfte darin begründet sein, daß die Parameter selbst keine konstanten Größen darstellen, sondern um einen gewissen Mittelwert schwanken, wobei die Abweichungen für alle Meßwerte prozentual die gleichen sind.

Bei der elektronischen Berechnung wurden natürliche Logarithmen eingesetzt. Es ist aus diesem Grunde

$$
a=-\frac{\log \mathrm{n}}{0,43429} \quad \text { und } b=\frac{\log \mathrm{m}}{0,43429}
$$

Den Berechnungen liegen die in den Tabellen III und XI von Krogir angegebenen Werte zugrunde und nicht die zum Teil in nicht ganz übersichtlicher Weise korrigierten Werte der Tabellen V und VII, die zur Aufstellung seiner Normalkurve dienten. Außerdem erfolgte eine Umrechnung der von ihm je Minute angegebenen Verbrauchswerte auf die Sauerstoffaufnahme je Stunde. Beim Goldfisch - Carassius auratus - (EgE und Krogh 1914) und den Tenebrio-Puppen wurden überdies seine Angaben für den Sauerstoffverbrauch je $\mathrm{kg}$ auf das Körpergewicht von $9,3 \mathrm{~g}$ bzw. 0,163 $\mathrm{g}$ umgerechnet. Ferner wurden einzelne Werte, die bei gleicher oder nahezu gleicher Temperatur starke Differenzen zeigten, in Anbetracht ihrer Unsicherheit, nicht zur Berechnung herangezogen. Diese Meßwerte sind aber natürlich in den Kurvendarstellungen und Tabellenwerten aufgenommen.

Im Folgenden sollen kurz die Ergebnisse besprochen werden, die sich bei der mathematischen Analyse der einzelnen von KROGH gegebenen Beispiele nach Gleichung (3) ergaben:

1. Enthirnte Kröte, 34,3 g - Tab. 1, Abb. 1-KroGH, Nr. 1.

Die enthirnte Kröte sollte wegen des Fehlens einer Reaktion auf äußere Reize, ohne daß Narkotika verwendet wurden, ein besonders günstiges Objekt sein. Trotzdem ergaben sich für praktisch die gleichen Temperaturen von $13,6 \mathrm{bzw}, 13,7^{\circ} \mathrm{C}$ drei verschiedene, um fast $20 \%$ differierende Werte. Hierdurch ist eine zuverlässige Bestimmung von $\mathrm{z}$ ausgeschlossen. Berücksichtigt man alle Werte, ergibt sich z zu $-70,5^{\circ} \mathrm{C}$. Die Abweichung

Tabelle 1

Enthirnte Kröte (Krogr 1914, Tab. III/1)

\begin{tabular}{cccc}
\hline $\begin{array}{c}\text { Tempe- } \\
\text { ratur }\end{array}$ & $\begin{array}{c}\text { Meßwerte } \\
02 \text { Verbr. } \\
\text { in }{ }^{\circ} \mathrm{C}\end{array}$ & $\begin{array}{c}\text { Berechnete Werte } \\
0 \mathrm{z} \text {-Verbr. } \mathrm{ml} / \mathrm{h}\end{array}$ & $\begin{array}{c}\text { Abweichung } \\
\text { in } \%\end{array}$ \\
& & $\mathrm{z}=-70,5$ & \\
& & $\log \mathrm{n}=328,7$ & \\
$\mathrm{~m}=4262,3$ & $-0,58$ \\
4,2 & 0,171 & 0,170 & $-0,74$ \\
7,7 & 0,270 & 0,268 & $+12,10$ \\
13,6 & 0,471 & 0,528 & $+2,33$ \\
13,6 & 0,516 & 0,528 & $-9,18$ \\
20,15 & 0,588 & 0,534 & $-4,71$ \\
27,15 & 1,062 & 1,012 & $+1,88$
\end{tabular}


von den gemessenen Werten überschreitet nur in zwei Fällen $5 \%$, liegt sonst weit darunter. Damit liegen die berechneten Werte noch in dem Schwankungsbereich, mit dem man bei Atmungsmessungen rechnen muß.

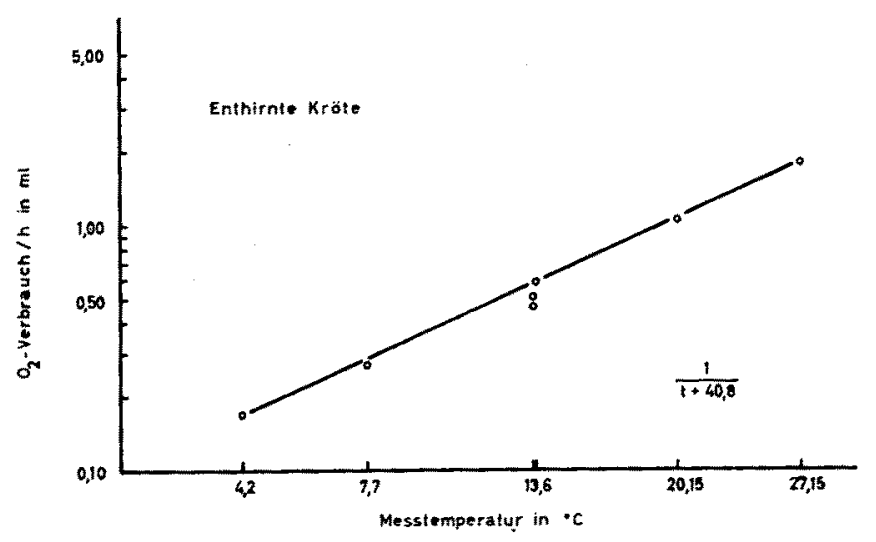

Abb. 1. Atmung der enthirnten Kröte als Funktion von $1 / t+40,8$

Ich persönlich halte aber den Wert $-40,8^{\circ} \mathrm{C}$ für zutreffender, den man erhält, wenn man die zweifelhaften drei Werte bei der Berechnung ausläßt, auch wenn dann einige andere Werte eine etwas stärkere Abweichung zeigen.

2. Mit Urethan narkotisierter Frosch, $30 \mathrm{~g}$ - Tab. 2, Abb. 2 - Krogh, Nr. 2. Diese Versuchsreihe ist dadurch von besonderem Interesse, da Krogr von ihr sagt: „Dieses Beispiel benötigt keine Reduktion und darf daher als das Zuverlässigste betrachtet werden." Aus dem Rahmen der übrigen Meßwerte fällt der Wert für $20,15^{\circ} \mathrm{C}$ - auch in der graphischen Darstellung von KroGH - Berücksichtigt man ihn, kommt man zu einem Wert für $z$ von $-35,2^{\circ} \mathrm{C}$, läßt man ihn bei der Berechnung aus, ergibt sich für $z$ der Wert $-32,7^{\circ}$ C. Die Differenz ist, wie man sieht, nicht sehr groß. Der abweichende Wert für $20,15^{\circ} \mathrm{C}$ läßt sich bei beiden $z$-Werten nicht einordnen. Mit Abweichungen seiner Größenordnung muß man gelegentlich

Tabelle 2

Urethan-narkotisierter Frosch, $30 \mathrm{~g}$ (KROGH 1914, Tab. III/2)

\begin{tabular}{|c|c|c|c|c|c|c|}
\hline \multirow[t]{2}{*}{$\begin{array}{l}\text { Tempe- } \\
\text { ratur } \\
\text { in }{ }^{\circ} \mathrm{C}\end{array}$} & \multirow[t]{2}{*}{$\begin{array}{c}\text { Meßreerte } \\
0_{2} \text {-Verbr. } \\
\mathrm{ml} / \mathrm{h}\end{array}$} & \multicolumn{3}{|c|}{$\begin{array}{c}\text { Berechnete Werte } \\
0_{2} \text {-Verbr. } \mathrm{ml} / \mathrm{h}\end{array}$} & \multicolumn{2}{|c|}{$\begin{array}{l}\text { Abweichung } \\
\text { in } \%\end{array}$} \\
\hline & & $\begin{aligned} z & = \\
\log n & = \\
m & =\end{aligned}$ & $\begin{array}{l}A \\
=-35,2 \\
=103,0 \\
=171,69\end{array}$ & $\begin{array}{c}\mathbf{B} \\
z=-32,7 \\
\log \mathrm{n}=15,2 \\
\mathrm{~m}=155,59\end{array}$ & $A$ & $B$ \\
\hline 4,2 & 0,414 & & 0,416 & 0,410 & $+0,48$ & $-0,97$ \\
\hline 7,7 & 0,672 & & 0,680 & 0,686 & $+1,19$ & $\begin{array}{l}+2,08 \\
+\end{array}$ \\
\hline 13,6 & 1,332 & & 1,328 & 1,369 & $-0,30$ & $+2,78$ \\
\hline 13,6 & 1,380 & & 1,328 & 1,369 & $-3,77$ & $-0,80$ \\
\hline 13,7 & 1,392 & & 1,341 & 1,383 & $-3,66$ & $-0,65$ \\
\hline 20,15 & 2,130 & & 2,361 & 2,458 & $+10,85$ & $+15,40$ \\
\hline 27,15 & 3,984 & & 3,820 & 3,996 & $-4,12$ & $+0,30$ \\
\hline
\end{tabular}




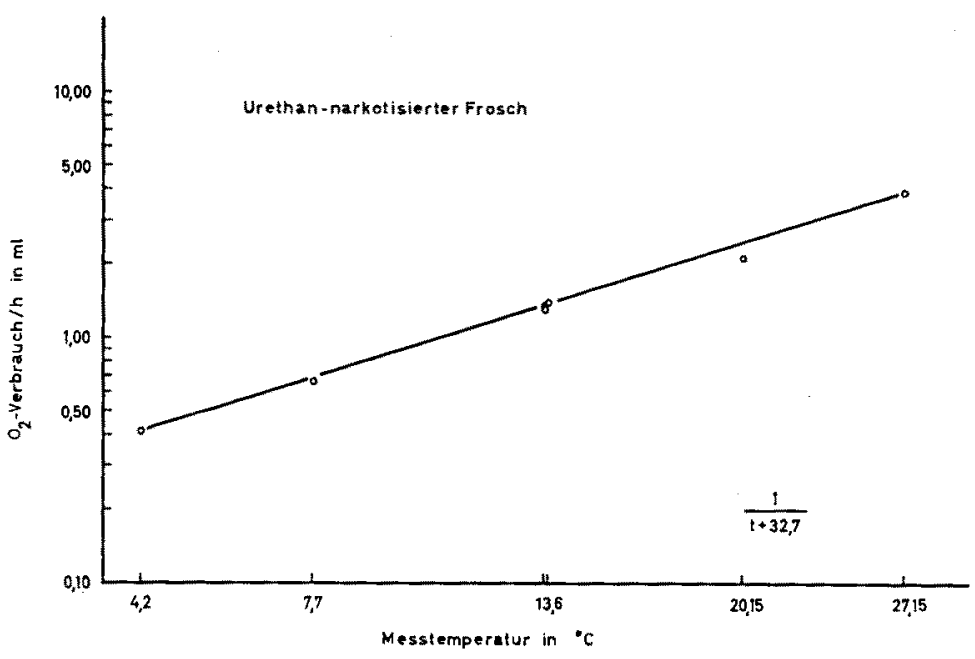

Abb. 2. Atmung eines narkotisierten Frosches als Funktion von $1 / t+32,7$

bei Atmungsmessungen rechnen. Die übrigen Werte lassen sich aber gut durch Gleichung (3) darstellen.

3. Curarisierter Frosch, etwa 35 g - Tab. 3, Abb. 3 - Krogh, Nr. 3.

Auch in diesem Fall weisen die Atmungsgrößen bei $15^{\circ} \mathrm{C}$ sehr große Unterschiede auf, die eine zuverlässige Bestimmung von $\mathrm{z}$ unmöglich machen. Werden der Berechnung alle Werte zugrunde gelegt, ergibt sich ein Wert für z von $-100,1^{\circ} \mathrm{C}$. Da aber offensichtlich die Angaben für $15,05^{\circ} \mathrm{C}$ und $15,1^{\circ} \mathrm{C}$, wie die Atmungsverminderung zeigt, nicht eindeutig sind, möchte ich den Wert für $z=-37,2$ für zuverlässiger halten, den man erhält, wenn man die beiden zweifelhaften Angaben nicht berücksichtigt. Dieser $z$-Wert stimmt dann auch größenordnungsmäßig mit dem vorangegangenen Beispiel überein. Daß keine vollständige Deckung der $z$-Werte zustande kommt, darf man vielleicht auf die Immobilisierung der Versuchsobjekte mit Curare zurückführen, die nicht wirklich gleichmäßig durchgeführt werden kann und auch im Verlauf der Versuche abklingt.

Tabelle 3

Curarisierter Frosch (Krogh 1914, Tab. III/3)

\begin{tabular}{cccc}
\hline $\begin{array}{c}\text { Tempe- } \\
\text { ratur } \\
\text { in }{ }^{\circ} \mathrm{C}\end{array}$ & $\begin{array}{c}\text { Meßrerte } \\
0_{2} \text {-Verbr. } \\
\mathrm{ml} / \mathrm{h}\end{array}$ & $\begin{array}{c}\text { Berechnete } \\
\text { 0z-Verbr. } \mathrm{ml} / \mathrm{h}\end{array}$ & $\begin{array}{c}\text { Abweichung } \\
\text { in } \%\end{array}$ \\
\hline & & $\mathrm{z}=-37,2$ & \\
\hline & & $\begin{array}{r}\log \mathrm{n}=101,8 \\
\mathrm{~m}=137,75\end{array}$ & $-1,63$ \\
\hline 5,95 & 0,612 & 0,602 & $+3,45$ \\
9,4 & 0,870 & 0,900 & $+10,14$ \\
15,05 & 1,410 & 1,553 & $-1,45$ \\
15,1 & 1,296 & 1,561 & $-1,70$ \\
15,2 & 1,596 & 1,571 & $+3,57$ \\
20,15 & 2,352 & 2,312 & $-2,06$ \\
24,75 & 3,024 & 3,132 & \\
24,75 & 3,198 & 3,132 &
\end{tabular}




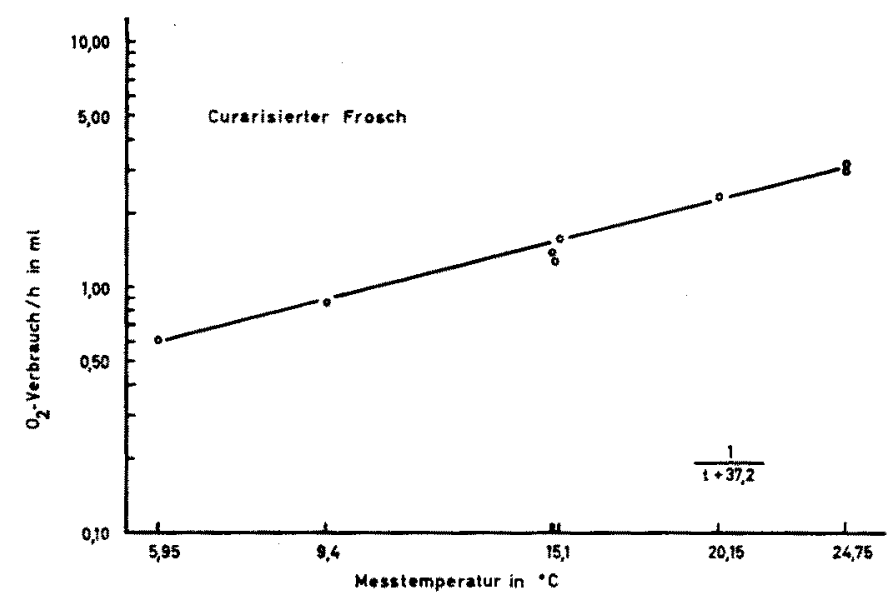

Abb. 3. Atmung eines curarisierten Frosches als Funktion von $1 / t+37,2$

4. Unvollständig mit Urethan narkotisierter Frosch, $40 \mathrm{~g}$ - Abb. 4 KROGH, Nr. 5 .

Die Gefahren, welche die Anwendung einer Narkose bei Atmungsmessungen bietet, zeigen die Beispiele 5 und 6 von unvollständig mit Urethan narkotisierten Fröschen. Die beiden Beispiele 2 und 3 ergaben - in den gegebenen Grenzen - eine befriedigende Übereinstimmung, nicht nur für

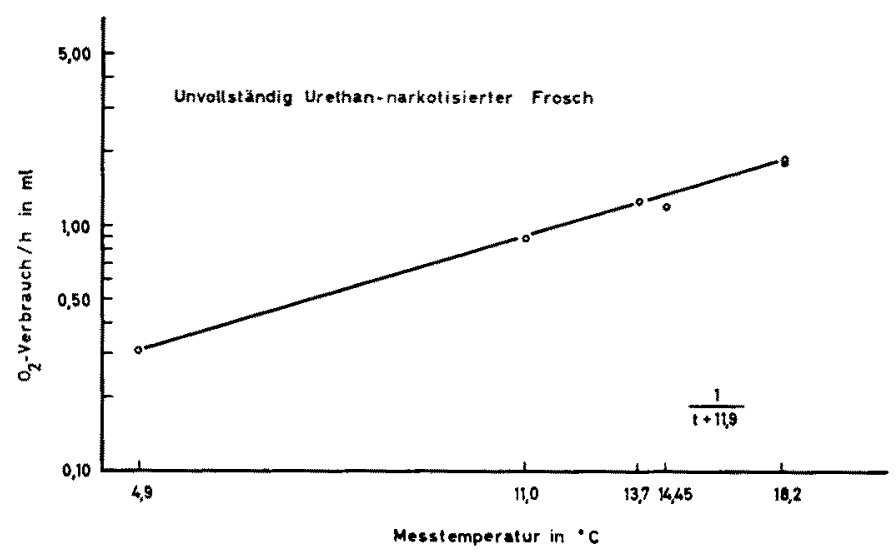

Abb. 4. Atmung eines unvollständig curarisierten Frosches als Funktion von $1 / t+11,6$

die Nullpunkttemperatur z, sondern auch für die beiden anderen Parameter $m$ und $\log n$. Demgegenüber ergaben sich bei der unvollständigen Urethan-Narkose sehr starke Differenzen. Für das Beispiel 5 von Krogh errechnet sich ein $z$-Wert von $-11,6^{\circ} \mathrm{C}$, wenn man den ganz offensichtlich aus dem Rahmen fallenden Wert für die Versuchstemperatur von $14,45^{\circ} \mathrm{C}$ ausläßt. Die übrigen Messungen ordnen sich sehr gut den berechneten Werten ein.

5. Unvollständig mit Urethan narkotisierter Frosch, $30 \mathrm{~g}$ - Abb. 5 KROGH, Nr. 6.

Grundsätzlich lassen sich die Meßwerte auch in diesem Falle befriedigend 


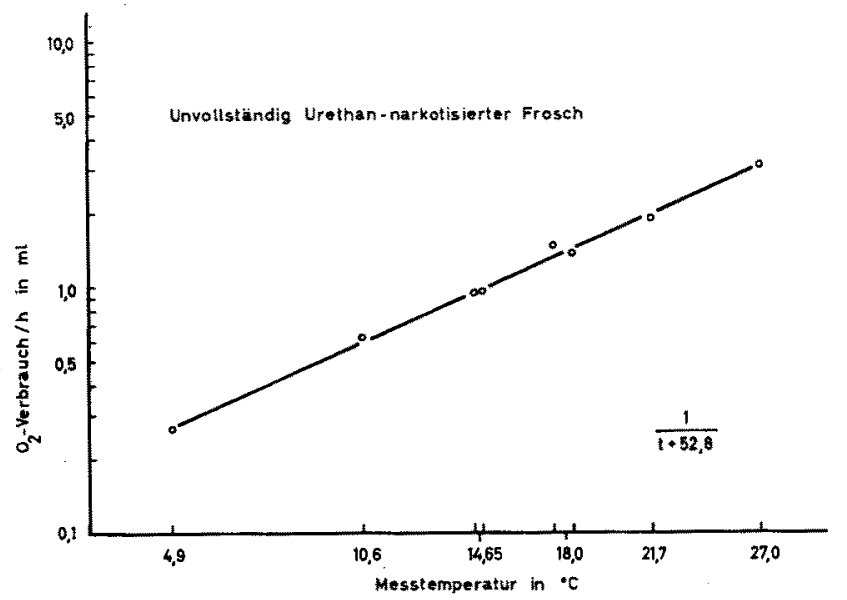

Abb. 5. Atmung eines unvollständig curarisierten Frosches als Funktion von $1 / t+52,8$

durch Gleichung (3) darstellen, jedoch mit dem wesentlich tieferen $z$-Wert von $-52,8^{\circ} \mathrm{C}$. Krogh hat die beiden Beispiele 5 und 6 , die sich offensichtlich nicht gut den übrigen Werten einfügen ließen, weder bei der Berechnung der Normalkurve berücksichtigt, noch sie in der graphischen Darstellung (Abb. 5 seiner Arbeit) aufgenommen.

6. Mit Curare immobilisierter Hund - Tab. 4, Abb. 6 - Krogh, Tab. IX. Die Zahlen von Krogr für den immobilisierten Hund weisen auch für gleiche Temperaturen erhebliche Streuung auf. Das ist angesichts der technisch kaum ganz einwandfrei durchzuführenden Messungen nicht verwunderlich. Trotzdem gelingt es mit einem $z$-Wert von $-10,4^{\circ} \mathrm{C}$ die Meßwerte in guter Näherung, entsprechend Gleichung (3), darzustellen. Wenn es Krogh glückte, trotz des niederen $z$-Wertes, seine Versuchszahlen mit der Normalkurve zu vereinbaren, so war das wohl nur

Tabelle 4

Immobilisierter Hund (Krogh 1914, Tab. IX)

\begin{tabular}{|c|c|c|c|}
\hline $\begin{array}{l}\text { Tempe- } \\
\text { ratur } \\
\text { in }{ }^{\circ} \mathrm{C}\end{array}$ & $\begin{array}{c}\text { Meßrevte } \\
0_{2} \text { Verbr. } \\
\mathrm{ml} / \mathrm{h}\end{array}$ & $\begin{array}{c}\text { Berechnete Werte } \\
0_{2}-\text { Verbr. } \mathrm{ml} / \mathrm{h}\end{array}$ & $\begin{array}{l}\text { Abweichung } \\
\text { in } \%\end{array}$ \\
\hline & & $\begin{aligned} z & =-10,4 \\
\log n & =36,2 \\
m & =64,04\end{aligned}$ & \\
\hline 14,1 & 2,15 & 2,12 & $-1,40$ \\
\hline 22,65 & 4,8 & 5,12 & $+6,67$ \\
\hline 28,0 & 7,8 & 7,29 & $-6,54$ \\
\hline 28,1 & 7,5 & 7,33 & $-2,27$ \\
\hline 28,65 & 6,8 & 7,56 & $+11,18$ \\
\hline 32,25 & 9,8 & 9,05 & $-7,65$ \\
\hline 36,75 & 11,4 & 10,91 & $-4,30$ \\
\hline 36,8 & 11,0 & 10,93 & $-0,64$ \\
\hline 36,85 & 10,3 & 10,95 & $+6,31$ \\
\hline 37,2 & 13,0 & 11,09 & $-14,69$ \\
\hline 39,65 & 11,2 & 12,09 & $+7,95$ \\
\hline 39,9 & 11,2 & 12,19 & $+8,84$ \\
\hline
\end{tabular}




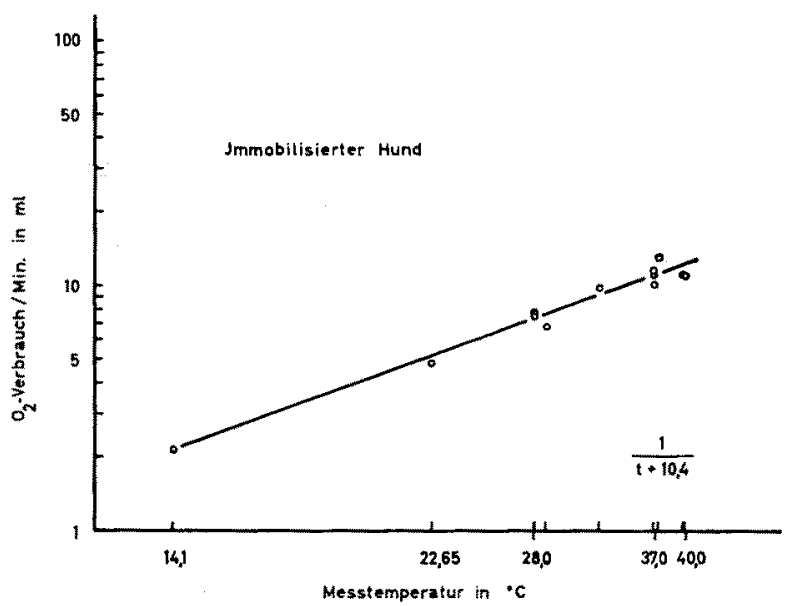

Abb. 6. Atmung eines mit Curare immobilisierten Hundes als Funktion von $1 / t+10,4$

dadurch möglich, daß er den Vergleich in Tab. X und der Kurve in Fig. 5 auf den engen Temperaturbereich zwischen $14^{\circ} \mathrm{C}$ und $28^{\circ} \mathrm{C}$ beschränkte, für den ihm die anderen Zahlen zur Verfügung standen. Den durch den niedrigen $z$-Wert angedeuteten andersartigen Kurvenverlauf hätte er bei höheren Temperaturen - wenn es biologisch möglich gewesen wäre - mit den Werten der Kaltblüter sicher nicht mehr zur Deckung bringen können.

7. Carassius auratus - Tab. 5, Abb. 7 - KRoGH, Nr. $\left.8^{*}\right)$.

Für die Temperatur von $7,9^{\circ} \mathrm{C}$ des nicht narkotisierten Goldfisches sind

Tabelle 5

Carassius auratus (EGE und KROGH)

\begin{tabular}{|c|c|c|c|}
\hline $\begin{array}{l}\text { Tempe- } \\
\text { ratur } \\
\text { in }{ }^{\circ} \mathrm{C}\end{array}$ & $\begin{array}{c}\text { Meßterte } \\
\text { O2-Verbr. } \text {-Ver } \\
\mathrm{ml} / \mathrm{h} / 9,3 \mathrm{~g}\end{array}$ & $\begin{array}{l}\text { Berechnete Werte } \\
02-\text { Verbr. ml/h/9,3 } \mathrm{g}\end{array}$ & $\begin{array}{c}\text { Abweichung } \\
\text { in } 0 / 0\end{array}$ \\
\hline & & $\begin{aligned} \mathrm{z} & =-34,4 \\
\log \mathrm{n} & =116,0 \\
\mathrm{~m} & =142,49\end{aligned}$ & \\
\hline 0 & 0,062 & 0,061 & $-1,61$ \\
\hline 0 & 0,060 & 0,061 & $+1,67$ \\
\hline 7,9 & 0,263 & 0,260 & $-1,14$ \\
\hline 7,9 & 0,331 & 0,260 & $-21,45$ \\
\hline 14,4 & 0,608 & 0,601 & $-1,15$ \\
\hline 14,9 & 0,614 & 0,636 & $+3,58$ \\
\hline 15,0 & 0,675 & 0,643 & $-4,74$ \\
\hline 20,1 & 1,010 & 1,065 & $+5,45$ \\
\hline 20,1 & 1,038 & 1,065 & $+2,60$ \\
\hline 24,8 & 1,635 & 1,570 & $-3,98$ \\
\hline 25,5 & 1,646 & 1,655 & $+0,55$ \\
\hline 25,7 & 1,641 & 1,680 & $+2,38$ \\
\hline 25,7 & 1,735 & 1,680 & $-3,17$ \\
\hline
\end{tabular}

Anmerkung: Die Atmungswerte von EGE und Krogr liegen höher als die in späteren Versuchen am Goldfisch gefundenen Zahlen, die in diesem Zusammenhang aber nicht berücksichtigt werden sollen. Die neueren Arbeiten über die Temperaturabhängigkeit der GoldfischAtmung finden sich in den: Verh. d. D. Zool. Ges., Wien 1962. 
zwei sehr unterschiedliche Meßwerte angegeben. Läßt man den höheren dieser beiden Werte unberücksichtigt, so erhält $z$ den Wert $-34,4^{\circ} \mathrm{C}$, mit ihm lassen sich alle anderen Versuchszahlen sehr gut vereinbaren. Wenn man den ausgelassenen Wert zur Berechnung heranzieht, erhält man einen $\mathrm{z}$-Wert von $-28,9^{\circ} \mathrm{C}$, wodurch die Abweichungen der übrigen Werte aber beträchtlich wachsen, ohne daß dieser aus dem Rahmen fallende Wert sich wesentlich besser einfügen läßt. Die Parameter $\log \mathbf{n}$ und $\mathrm{z}$ dieses Objektes sind größenordnungsmäßig übereinstimmend mit denen

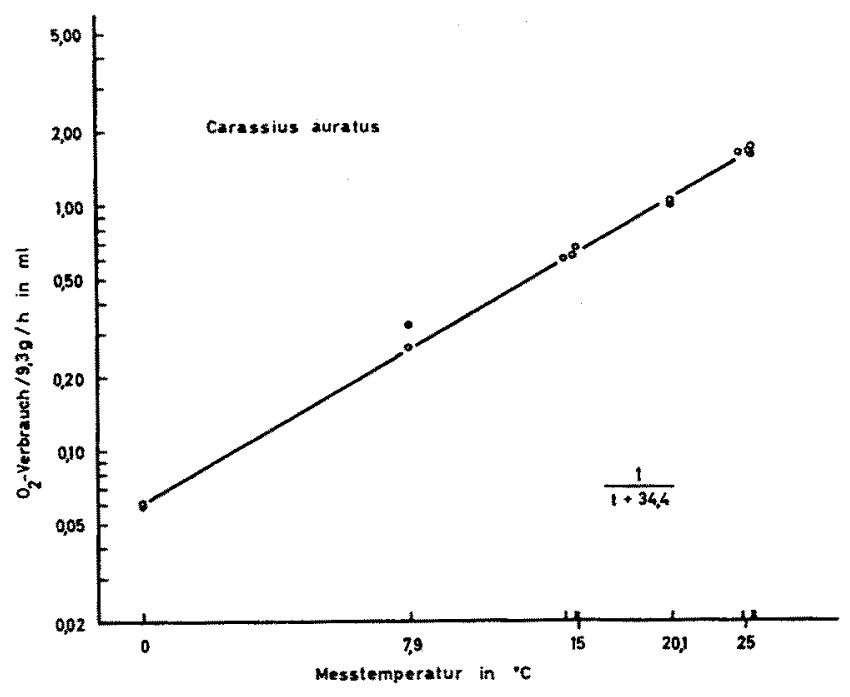

Abb. 7. Atmung eines Goldfisches als Funktion von $1 / t+34,4$

der Beispiele 2 und 3. Es ist daher nicht erstaunlich, daß sie sich sehr gut der Normalkurve einfügen. Die Werte des mit Urethan narkotisierten Fisches folgen etwa denen des normalen Fisches, umfassen aber eine wesentlich geringere Temperaturspanne, enthalten außerdem für die Temperatur von 19,1 bzw. 19,2 $\mathrm{C}$ stark aus dem Rahmen fallende Zahlen. Eine sichere Festlegung von $\mathrm{z}$ ist unter diesen Umständen nicht möglich. Berücksichtigt man die $19^{\circ}$-Werte nicht, kommt man für $\mathrm{z}$ auf $-39,2^{\circ} \mathrm{C}$ und

Tabelle 6

Puppen von Tenebrio (KroGH 1914, Tab. XI)

\begin{tabular}{|c|c|c|c|}
\hline $\begin{array}{l}\text { Tempe- } \\
\text { ratur } \\
\text { in }{ }^{\circ} \mathrm{C}\end{array}$ & $\begin{array}{l}\text { Meßzwerte } \\
0 \mathrm{2}-\text { Verbr. } \\
\mathrm{ml} / 0,163 \mathrm{~g} / \mathrm{h}\end{array}$ & $\begin{array}{c}\text { Berechnete Werte } \\
0_{2}-\text { Verbr. } \\
\mathrm{ml} / 0,163 \mathrm{~g} / \mathrm{h}\end{array}$ & $\begin{array}{c}\text { Abweichung } \\
\text { in } \%\end{array}$ \\
\hline & & $\begin{aligned} \mathrm{z} & =-18,9 \\
\log \mathbf{n} & =71,2 \\
\mathbf{m} & =2,07\end{aligned}$ & \\
\hline 10 & 0,00709 & 0,00715 & $+0,85$ \\
\hline 15 & 0,01695 & 0,01649 & $-2,71$ \\
\hline 20 & 0,03016 & 0,03067 & $+1,69$ \\
\hline 25 & 0,04890 & 0,04953 & $+1,29$ \\
\hline 30 & 0,07254 & 0,07253 & $-0,01$ \\
\hline 32.5 & 0.08623 & 0,08536 & $-1,01$ \\
\hline
\end{tabular}


für $\log$ n auf 119,94 . Bei diesem Objekt scheint der Einfluß der UrethanNarkose auf die Atmung nicht so stark gewesen zu sein, wie bei den Beispielen 4 und 5. Für eine genauere Analyse sind die zahlenmäßigen Unterlagen nicht ausreichend.

8. Tenebrio-Puppen - Tab. 6, Abb. 8 - KRoGH, Tab. XI.

$\mathrm{Zu}$ den für dieses Objekt gefundenen Zahlen sagt KroGH: „Die Kurve für die Puppen muß hinsichtlich der absoluten Übereinstimmung aller Messungen als außergewöhnlich zuverlässig angesehen werden..." Aber die

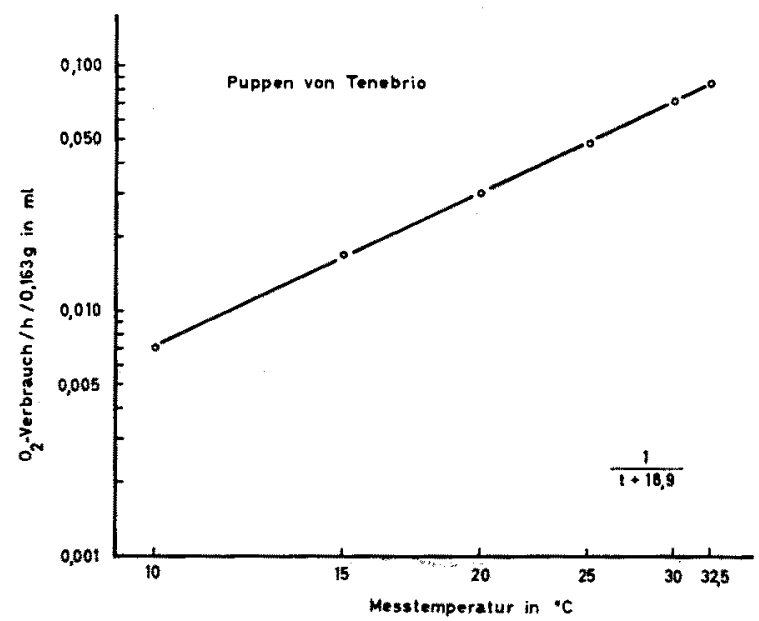

Abb. 8. Atmung von Tenebrio-Puppen als Funktion von $1 / t+18,9$

Atmungswerte ließen sich nicht mit seiner Normalkurve zur Deckung bringen. Krogh fährt dann fort: „... die Kurven müssen daher als ganz abweichend von dem angesehen werden, was, soweit bis jetzt bekannt, die Beziehung zwischen Temperatur und Stoff wechsel bei Vertebraten wiedergibt."

Dieses Beispiel zeigt besonders deutlich den sehr viel weiteren Umfang, in dem Gleichung (3) anwendbar ist, denn die Meßwerte der TenebrioPuppen lassen sich außerordentlich gut darstellen, wenn man als $z$-Wert $-18,9^{\circ} \mathrm{C}$ wählt. Die hervorragende Übereinstimmung zwischen Berechnung und Versuchsergebnis bestätigen das Urteil, das KroGH über diese Messungen fällte und die Präzision der Zahlenangaben läßt eine eindeutige mathematische Auswertung zu.

Zusammenfassend kann man also feststellen, daß die der zitierten Arbeit von KroGH entnommenen Meßwerte sich mit zum Teil sehr guter Näherung durch eine einheitlich mathematische Funktion darstellen lassen. Hiermit ist die Hoffnung, die KroGH damals aussprach (Seite 505) erfüllt: "Q $Q_{10}$ ist weit davon entfernt konstant zu sein ... und der offensichtliche Schluß ist der, daß die Stoffwechselprozesse von Tieren im ganzen genommen, nicht der Regel von VAN'T Hoff folgen, aber daß es möglich sein wird, irgendeinen anderen Ausdruck für sie zu finden." Hierbei muß man allerdings die Einschränkung machen, daß trotz aller von Krogh aufgewandten Mühe, seine Versuchszahlen nicht immer die Sicherheit bieten, die eine mathematische Analyse erfordert. Hierfür wäre eine größere Zahl von Versuchsreihen erforderlich gewesen, als sie KrogH durchführte. 
Während also im allgemeinen sich die Temperaturfunktion der Atmung der einzelnen Versuchsobjekte recht befriedigend durch Gleichung (3) wiedergeben läßt, gilt das gleiche nicht für die Zahlenwerte, die KRoGH in seiner Tab. VII für die Normalkurve gibt. In Abb. 9 sind sie als Funktion von $1 / t+30$ dargestellt. Wie man aus der graphischen Darstellung ersieht, liegen die Werte unterhalb $8^{\circ} \mathrm{C}$ etwas über dem berechneten Wert, oberhalb $8^{\circ} \mathrm{C}$ dagegen in der Mehrzahl tiefer. Die der Normalkurve zugrunde liegenden Zahlenwerte sind auch mit anderen $z$-Werten nicht mit gleicher Genauigkeit durch Gleichung (3) wiederzugeben. Das kann nicht erstaunen, da KroGH seine Normalkurve als Mittelwert aus den verschiedenen oben angeführten Beispie-

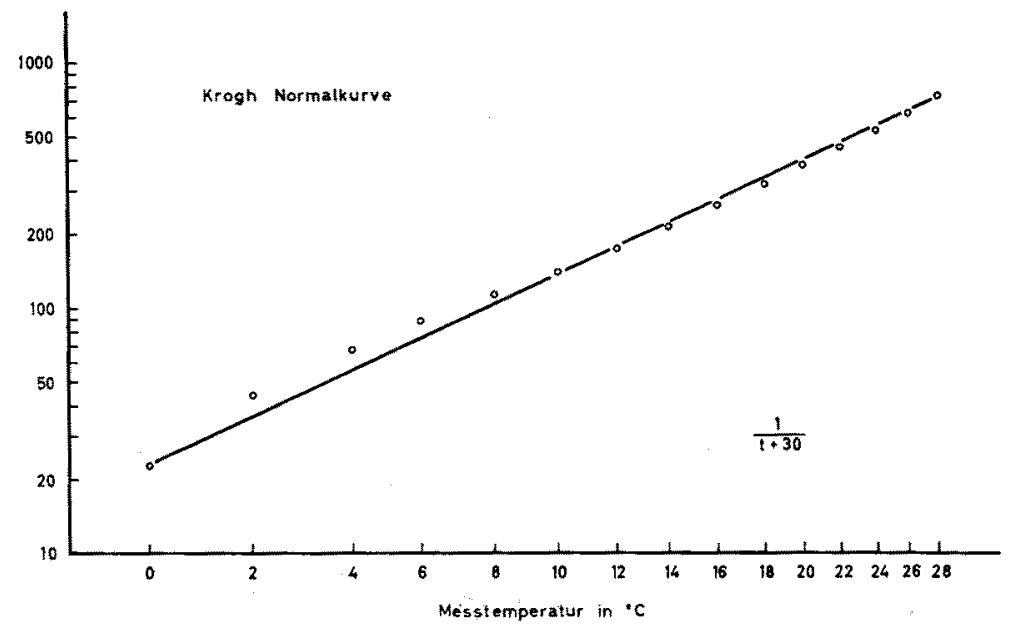

Abb. 9. Zahlenwerte der Normalkurve von KROGH im Vergleich mit der Funktion $1 / \mathrm{t}+30$

len berechnete. Dabei handelt es sich aber bei den auf diese Weise zusammengefaßten Kurven, wie wir oben sahen, um Kurven mit sehr unterschiedlichen Parametern, also verschiedenem Verlauf. Eine solche Mittelwertbildung konnte also nicht zu einer auf der gleichen Basis berechneten Kurve dienen, auch wenn die einzelnen Kurven Gleichung (3) folgen. Aus diesem Grunde verzichtete ich auf eine genauere Berechnung des $z$-Wertes der Krogh-Kurve und begnügte mich mit dem Näherungswert für z von $-30^{\circ} \mathrm{C}$, der in der Nähe der $z-W$ erte der wichtigsten Beispiele von KROGH liegt.

Die Angabe von exakten Zahlenwerten, entsprechend Gleichung (3), besitzt vor dem Kroghschen Verfahren eines einfachen Vergleichs mit einer nicht exakt definierten Kurve zahlreiche Vorzüge:

1. schließt der einfache Kurven-Vergleich erhebliche Fehlerquellen ein. Betrachtet man die von KRoGH aufgestellten Kurven, so kann von einer strengen Anordnung seiner Meßwerte auf derselben keine Rede sein. Bei der starken Streuung der Punkte ist die Entscheidung darüber, ob man sie noch mit ihr als zusammenfallend bezeichnet, stark subjektiven Momenten unterworfen,

2. können die Besonderheiten jeden Kurvenverlaufs nach Gleichung (3) zahlenmäßig ausgedrückt werden und die Parameter gestatten einen exakten Vergleich zwischen verschiedenen Kurven,

3. ist die Forderung der Gleichung (3), daß die Meßpunkte möglichst genau 
auf einer Geraden liegen müssen, sehr viel leichter einer objektiven Kontrolle zugänglich,

4. gestattet Gleichung (3) in jedem Fall, Abweichungen von den theoretischen Zahlenwerten quantitativ zu bestimmen,

5. ist es möglich, mit Gleichung (3) Temperaturfunktionen zu erfassen, die sich mit der Normalkurve nicht mehr vereinbaren lassen.

\section{Die Temperaturfunktion von JøRGENSEN}

Zur mathematischen Darstellung der Kroghschen Normalkurve hat JøRGENSEN (1916) - zitiert nach NIELSEN und EvANS (1960) - einen grundsätzlich anderen Vorschlag gemacht, von dem allerdings in der Literatur, trotz seiner offensichtlichen Eignung, kein Gebrauch gemacht wurde. Er fand, daß die KroGHsche Normalkurve dargestellt werden kann durch die Gleichung:

$$
y=a+b \cdot c^{t}
$$

$\mathrm{y}=$ Sauerstoff verbrauch, $\mathrm{t}=$ Temperatur in ${ }^{\circ} \mathrm{C} ; \mathrm{a}, \mathrm{b}$ und $\mathrm{c}=$ Konstanten. Diese Gleichung entspricht in ihrem Grundzug der Berthelot-Formel (1), wie man leicht erkennt, wenn man a auf die linke Seite der Gleichung bringt

$$
(y-a)=b \cdot c^{t}
$$

In der graphischen Darstellung im semilogarithmischen Koordinatensystem stellt die Gleichung eine Tangente der Kurve dar, die so gewählt ist, daß für alle Temperaturen der Abstand der Meßpunkte von der Tangente gleich a ist. Die Parameter, die diese Forderung erfüllen, lassen sich relativ leicht rechnerisch ermitteln.

Nielsen und Evans benutzten die Formel von Jørgensen zur Darstellung ihrer sehr exakten Zahlen über die Abhängigkeit der Entwicklungsgeschwindigkeit der Mücke Aedes taeniorhynchus von der Temperatur und konnten eine überraschend gute Übereinstimmung erreichen. Ich selbst habe mich an den sehr zuverlässigen Zahlen der Untersuchung von Јов auch von der Brauchbarkeit der Formulierung von JørGENSEN überzeugen können.

Allerdings stellt die Formel von JørGENSEN eine rein mathematische Lösung des Problems dar. Die Unterteilung des erfaßten Temperaturbereiches in wenigstens zwei gleichgroße Intervalle erreicht die Ausrichtung der Parameter nach drei Meßwerten im Minimum. Es ist verständlich, daß innerhalb des sehr eingeengten Temperaturbereiches der Intervalle eine einfache Exponentialfunktion den dazwischenliegenden Meßpunkten zumindest sehr nahekommt.

Der Nachteil der Formulierung von Jørgensen liegt darin, daß sie im Grunde genommen die Gleichung einer Geraden als Funktion der Temperatur wiedergibt, von der die Meßwerte nur einen gewissen Abstand haben, auf der sie selbst aber nicht liegen. Daher dürfte es auch schwierig sein, den drei in ihr enthaltenen Parametern eine biologische Deutung zu geben, was bei Gleichung (3) wenigstens in gewissem Umfang möglich ist. Es ist dieses ein Bedenken, auf das auch NiELSEN und Evans hinweisen. Außerdem würde nach der mathematischen Form der Gleichung (4) der Stoffwechsel oder die Entwicklungsgeschwindigkeit einen von der Temperatur unabhängigen Anteil - nämlich a - haben. Diese Annahme ist aber sehr unwahrscheinlich.

Die Daten von Nielsen und Evans schließen die Schwierigkeit ein, daß die Entwicklungsgeschwindigkeit ihrer Versuchstiere bei Temperaturen oberhalb $28,5^{\circ} \mathrm{C}$ nicht mehr so schnell zunimmt, wie bei tieferen Temperaturen. 
Die Autoren korrigieren diesen Effekt durch Einführung eines Korrekturgliedes, das ebenfalls der Formel von Jørgensen entspricht, aber entgegengesetztes Vorzeichen hat. Sie erreichen auf diese Weise im ganzen Temperaturbereich eine hervorragende Ubereinstimmung ihrer berechneten Werte mit den Versuchszahlen.

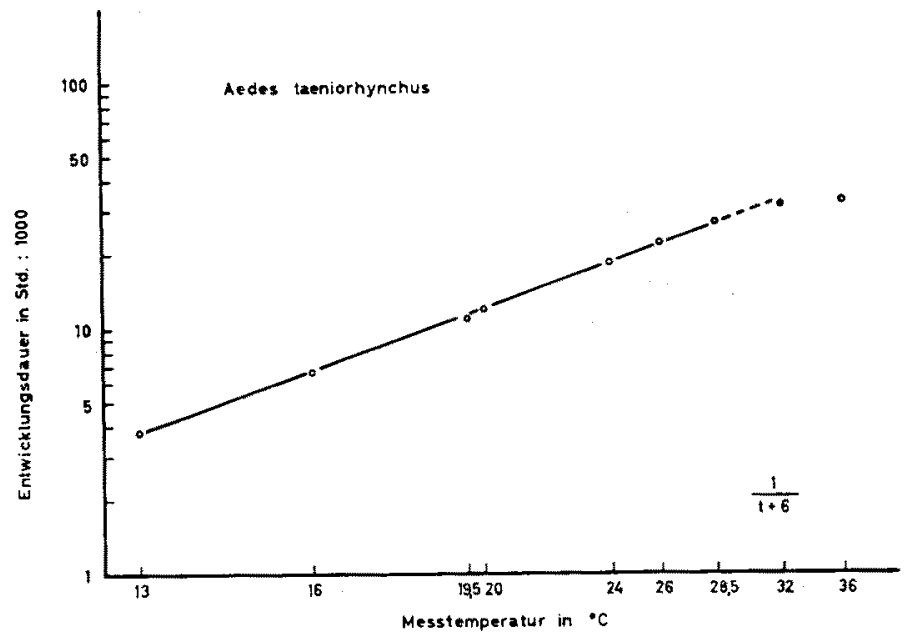

Abb. 10. Entwicklungsgeschwindigkeit von Mückenlarven als Funktion von $1 / t+6$

Mein Versuch, die Zahlenwerte für die Entwicklungsgeschwindigkeit der Aedes-Puppen nach Gleichung (3) darzustellen, lieferte auch nicht für den ganzen Temperaturbereich ein befriedigendes Ergebnis (Abb. 10). Ich habe aber aus folgenden Erwägungen davon Abstand genommen, durch eine mehr oder minder willkürliche Korrekturformel den Anschluß an alle Versuchswerte zu erreichen.

\section{Tabelle 7}

Vergleich der Berechnung der Versuchszahlen von Nielsen und Evans nach Jørgensen und nach Gleichung (3)

\begin{tabular}{|c|c|c|c|}
\hline $\begin{array}{l}\text { Tempe- } \\
\text { ratur } \\
\text { in }{ }^{\circ} \mathrm{C}\end{array}$ & $\begin{array}{c}\text { Versuchswerte } \\
\text { Entwicklungs- } \\
\text { Geschwindigkeit } \\
1000 \\
\text { h }\end{array}$ & $\begin{array}{l}\text { nach JORGENSEN } \\
y=a+b \cdot c\end{array}$ & $\begin{aligned} & \text { nach Gleichung }(3) \\
& \mathrm{z}=-6 \\
& \log \mathrm{n}=36,8 \\
& \mathrm{~m}=313,1\end{aligned}$ \\
\hline 13,0 & 3,78 & 3,74 & 3,62 \\
\hline 16,0 & 6,72 & 6,81 & 6,65 \\
\hline 19,5 & 11,04 & 11,20 & 11,28 \\
\hline 20,0 & 12,06 & 11,90 & 12,03 \\
\hline 24,0 & 18,64 & 18,41 & 18,58 \\
\hline 26,0 & 22,39 & 22,31 & 22,16 \\
\hline 28,5 & 26,95 & 27,90 & 26,84 \\
\hline 32,0 & 31,81 & 37,33 & 33,65 \\
\hline 36,0 & 32,97 & 50,90 & 41,63 \\
\hline
\end{tabular}

In den Fällen, in denen sich Gleichung (3) zur Darstellung der Temperaturfunktion geeignet erwies, handelte es sich um Messungen, bei denen die Versuchstiere - aus einer mehr oder minder konstanten Temperatur kommend - 
kurzfristig den Meßtemperaturen ausgesetzt werden. Man kann daher annehmen, daß die Versuchstiere auf der Basis eines konstanten physiologischen Zustandes auf die Temperaturveränderung reagierten. In den Versuchen über die Entwicklungsgeschwindigkeit sind die Organismen aber für längere Zeit konstanten Bedingungen ausgesetzt. Wie wir vor allem durch die Arbeiten von Precht (1.961) und seiner Schule wissen, kann in einem solchen Fall der physiologische Zustand der Organismen sich ändern, so daß die Stoffwechselgröße bei einer bestimmten Temperatur eine andere ist, als bei Tieren, die in einer anderen Temperatur gehalten wurden.

Die Untersuchungen von PrEchT und seinen Mitarbeitern haben Beweise dafür erbracht, daß die Temperatur-Adaptation z. B. auf Anderungen in der Fermentkonzentration beruht, die in Gleichung (3) vermutlich in dem Wert für $m$ enthalten ist. Die Erscheinung der Temperatur-Adaptation macht daher verständlich, daß die mathematische Darstellung der Temperaturabhängigkeit von Entwicklungsgeschwindigkeiten auf größere Schwierigkeiten stößt als die Temperaturabhängigkeit von Stoffwechselvorgängen, die in kurzfristigen Versuchen gemessen werden. Daher konnte vermutlich bis heute noch keine allgemeingültige Formulierung der Temperaturfunktion von Entwicklungsvorgängen aufgestellt werden. So möchte auch ich der Ansicht von Precht zustimmen, daß nicht anzunehmen ist, daß die Temperaturfunktion von Entwicklungsgeschwindigkeiten identisch mit der Temperaturfunktion von Stoffwechselvorgängen ist.

Wenn man diese Einschränkung in der Anwendung von Stoffwechselfunktionen auf Entwicklungsgeschwindigkeiten berücksichtigt, darf man sagen, daß die nach Gleichung (3) berechneten Werte in dem weiten Temperaturbereich zwischen $13^{\circ} \mathrm{C}$ und $28,5^{\circ} \mathrm{C}$ eine sehr gute Übereinstimmung mit den Versuchswerten zeigen (Tab. 7). Die Differenz wird etwas größer bei $32^{\circ} \mathrm{C}$ und fällt bei $36^{\circ} \mathrm{C}$ vollkommen aus den Grenzen einer zulässigen Abweichung. Der Geltungsbereich von Gleichung (3) erstreckt sich also über einen Temperaturbereich von mehr als $15^{\circ} \mathrm{C}$ und erweist sich der Formel von JørGensen etwas überlegen, die für $28,5^{\circ} \mathrm{C}$ ohne das Korrekturglied schon einen wesentlich zu hohen Wert ergibt und bei $32^{\circ} \mathrm{C}$ völlig versagt. In den gegebenen Grenzen scheint demnach Gleichung (3) auch für die mathematische Darstellung der Temperaturabhängigkeit von Entwicklungsgeschwindigkeiten geeignet zu sein.

\section{Diskussion}

Einer mathematischen Analyse biologischer Vorgänge sind in allen Fällen Grenzen gesetzt. Alle Lebensfunktionen laufen nur in einem mehr oder minder beschränkten Temperaturbereich normal ab. Überschreiten wir diese Grenzen, kommt es zu offensichtlichen Schädigungen, die mit dem Absterben des Organismus eindeutig sichtbar werden. Aber schon vorher lassen sich tiefergreifende Störungen erkennen, die zwar reversibel sein können, aber im Stoffwechsel sich dadurch z. B. äußern, daß die gemessenen Atmungswerte niedriger sind als bei tieferen Temperaturen. Ebenso wie NiELsen und Evans in ihren Versuchen, haben schon früher PüTter (1914), JANISCH (1927) und andere Autoren diese Erscheinung durch Reaktionsabläufe mit negativem Vorzeichen zu deuten versucht. Ich selbst möchte zunächst solche Kurvenabschnitte nicht berücksichtigen, da die in diesen Bereichen stark schwankenden Meßwerte für eine zahlenmäßige Analyse wenig geeignet sind und beschränke mich auf den als physio- 
logisch zu bezeichnenden Temperaturbereich, der von den Organismen ohne erkenntliche Störung ertragen wird. Für diesen Bereich ist aber von einer mathematischen Formulierung zu verlangen, daß sie über einen möglichst weiten Temperaturbereich die Versuchswerte durch konstante Kennwerte wiederzugeben gestattet.

Die Beschränkung gilt nicht nur für die oberen Grenztemperaturen, sondern auch vor allem für die niederen Temperaturen. In der Nähe des Gefrierpunktes sind im allgemeinen die Kurven stärker gekrümmt und dadurch werden in diesem Bereich an die mathematischen Formulierungen höhere Anforderungen gestellt als bei höheren Temperaturen, wo sich oft mit hinreichender Genauigkeit die Versuchswerte durch einfachere Exponentialfunktionen darstellen lassen.

Die zweite Begrenzung stellt die relativ große Streuung biologischer Meßwerte dar, die in keinem Falle eine Zuverlässigkeit von Messungen an unbelebten Systemen erreicht. Die technischen Schwierigkeiten, denen man auch heute noch immer wieder bei biologischen Messungen gegenübersteht, dürften mit fortschreitender Verbesserung der Untersuchungsverfahren geringer werden. Nicht zu vermeiden sind die biologischen Ursachen der Streuung der Meßwerte. Die Atmungsgröße eines Tieres ist von einer sehr großen Zahl von Faktoren bestimmt, von denen bis heute nur ein sehr kleiner Teil erkennbar ist. Man kann daher nicht immer mit Sicherheit erreichen, daß man mit Organismen im gleichen physiologischen Zustand arbeitet. Bei Tieren stört vor allem in diesem Zusammenhang solche Messungen die nicht zu kontrollierende Aktivität. Das von Krogh aus diesem Grunde eingeschlagene Verfahren, mit narkotisierten Tieren unter „Standard-Bedingungen“ zu arbeiten, setzt aber eine genaue Analyse der Wirkung der Narkotika voraus, die in nur wenigen Fällen gegeben ist.

Noch schwerwiegender und nicht auszuschalten sind wahrscheinlich vorhandene individuelle Unterschiede in der Fermentkonzentration, die ebenso wie alle übrigen biologischen Größen nicht eindeutig festgelegt sein dürfte, sondern in gewissem Umfang variiert. Es empfiehlt sich daher, die Temperaturabhängigkeit des Stoff wechsels an einzelnen Tieren im ganzen Temperaturbereich zu messen. Dadurch kann diese Störungsquelle ausgeschaltet werden, sie ist aber nicht zu vermeiden, wenn man mit Tieren unterschiedlicher Größe arbeitet.

Bei der aus den genannten Gründen unvermeidlich gegebenen Schwankungsbreite biologischer Meßwerte kann eine mathematische Analyse nur dann zum Erfolg führen, wenn man bei seinen Messungen möglichst weite Bereiche erfaßt, so daß die erfaßten Differenzen ein Vielfaches der gegebenen Schwankungsbreite der Meßwerte darstellen. Bei Temperaturfunktionen würde das heißen, daß man den ganzen Temperaturumfang erfaßt, der noch mit einer normalen Funktion des Organismus vereinbar ist. Bei der mathematischen Analyse der Größenabhängigkeit des Stoffwechsels von Tieren hat sich in der Zwischenzeit gezeigt, daß man auf diese Weise zu reproduzierbaren Zahlen kommen kann.

Gerade das letztgenannte Problem, dessen mathematische Analysierbarkeit in den beiden letzten Jahrzehnten durch zahlreiche Beispiele gesichert ist, zeigt, daß auch in dafür geeigneten Gebieten der Biologie die Anwendung der Mathematik wichtige Aufschlüsse vermitteln kann. Eine mathematische Darstellung gemäß der sogenannten allometrischen Formel: 


$$
y=a \cdot x^{b}
$$

gestattet durch Angabe der Werte für a und b, die Stoffwechselgröße y für alle Tierdimensionen eindeutig wiederzugeben. Die bis dahin übliche Wiedergabe von Stoffwechselwerten, bezogen auf die Gewichtseinheit, ist zur Charakterisierung der Stoffwechselgröße eines Organismus ungeeignet, da dieser Quotient von der Tiergröße abhängt.

Für die mathematische Darstellung der biologisch noch bedeutungsvolleren Temperaturfunktion wurde in der vorliegenden Untersuchung Gleichung (3) vorgeschlagen, deren Anwendbarkeit über die zitierten Fälle hinausgehend eine Reihe von Beispielen zeigte. Gleichung (3) dürfte demnach - wenn auch nicht in allen Fällen, so doch in weitem Umfang - geeignet sein, die Temperaturfunktion von Stoffwechselvorgängen darzustellen. Wie groß ihr Geltungsbereich ist, läßt sich heute noch nicht übersehen, da trotz des Vorliegens zahlreicher Untersuchungen über die Temperaturwirkung nur ein Bruchteil davon zur mathematischen Auswertung geeignet ist.

In den Fällen jedoch, in denen wir mit Hilfe von Gleichung (3) eine mathematische Analyse durchführen können, sind wir hierdurch in der Lage, den Temperatureinfluß eindeutig wiedergeben zu können. Die Bedeutung einer mathematischen Analyse ergab sich sehr bald, als bei der Verarbeitung der Versuchswerte von JoB, der in letzter Zeit verschiedentlich aufgezeigte Zusammenhang zwischen Tiergröße und Temperaturabhängigkeit zahlenmäßig sicherstellt und darüber hinausgehend näherungsweise mathematisch erfaßt werden konnte. Gleichung (3) schließt nämlich auch die Größenfunktion des Stoffwechsels dadurch ein, daß die Parameter $m, n$ und $z$ größenabhängig sind. Die beiden Funktionen sind nicht voneinander zu trennen, sondern nur gemeinsam zu behandeln.

Die Möglichkeit, die bei den Organismen zum Teil sehr stark variierende Sequenz der Meßwerte durch die Angabe der Parameter m, n und z wiederzugeben, dürfte den Weg zur Lösung zahlreicher anderer Probleme ebnen. Man kann hierbei an die Beziehungen dieser Größen zu den Milieubedingungen denken oder an eine exakte Beschreibung der Adaptationserscheinungen, um nur zwei naheliegende Fragestellungen zu nennen.

Bei der Bearbeitung aller dieser offenstehenden Probleme kann sich der Zwang, mathematisch analysierbare Werte zu erhalten, einmal vorteilhaft auf die künftige Entwicklung der Versuchstechnik auswirken. Außerdem bietet die mathematische Analyse eine Möglichkeit zur Kritik von Versuchswerten und hat, wie eigene Erfahrungen zeigten, verschiedentlich z. B. Rechenfehler aufgedeckt.

Zunächst stellt die mathematische Analyse der Temperaturfunktion biologischer Prozesse nur einen Versuch dar, die im Experiment gefundenen Zahlenbeziehungen durch eine geeignete Formel darzustellen. Wenn dieser Versuch sich als erfolgreich erweist, ist hierin schon ein wesentlicher Fortschritt zu sehen, da wir damit in die Lage versetzt sind, an die Stelle umständlicher Beschreibungen Kennzahlen zu setzen, die uns gestatten, Versuchsergebnisse in prägnanter und eindeutiger Weise wiederzugeben. Die Kennzahlen bilden dann für alle vergleichenden Untersuchungen exakte Unterlagen. Darüber hinaus ist aber zu erwarten, daß die Parameter der Gleichung (3) selbst wichtige Aussagen über die zu Grunde liegenden Zusammenhänge aufdecken werden. 
Von den Parametern der Gleichung (3) stellt m mathematisch die maximale Leistungsfähigkeit des Systems dar und enthält wahrscheinlich Fermentkonzentrationen. Dieser Schluß, der aus der Form der Gleichung hervorgeht, erfuhr eine unerwartete Bestätigung bei der Auswertung der Versuche von Joв, die zeigte, daß im Verlauf des Wachstums der Wert für $m$ annähernd mit der $2 / 3$ Potenz des Gewichtes zunimmt. Diese oberflächenproportionale Zunahme der Fermentkonzentration im Verlaufe des Wachstums ist in verschiedenen Untersuchungen auch experimentell bewiesen worden. Einer direkten Bestimmung ist der Wert für m nicht zugängig.

Durch die enge Verknüpfung der Parameter der Gleichung (3) wird $\mathrm{m}$ von der Größe von $n$ und $z$ beeinflußt und zwar wächst $m$ mit zunehmendem Wert von z. Nach den Erfahrungen an Salvelinus stellt m eine Exponentialfunktion des Tiergewichtes dar, die wir in den vorliegenden Fällen nicht kennen. Es ist daher nicht zulässig, $m$ durch eine einfache Division auf die Gewichtseinheit zu berechnen. Man kann aber daran denken, daß $\mathrm{m}$ audh in Zukunft sich als eine wichtige Basis für den temperatur- und gewichtsunabhängigen Vergleich der Atmungsgröße erweist. Anderungen des Wertes von $\mathrm{m}$ beeinflussen nicht die Temperaturfunktion als solche, sondern bewirken im semilogarithmischen Koordinatensystem nur eine Parallelverschiebung der Geraden, durch die wir unsere Meßwerte darstellen. In dieser Hinsicht war das Vorgehen von KroGH berechtigt, der durch Multiplikation mit geeigneten Faktoren seine Meßwerte mit der Normalkurve zur Deckung brachte.

Der eigentliche Verlauf der Temperaturfunktion wird ausgedrückt durch den Nenner $\mathbf{n}^{1 / t-z}$ der Gleichung (3). Mathematisch ist es der Anteil von m, der nicht an der Umsetzung beteiligt - also inaktiv — ist, während y den aktiven Anteil repräsentiert. Man erkennt leicht aus Gleichung (3), daß das Produkt von aktivem und inaktivem Anteil konstant, nämlich gleich $\mathrm{m}$, ist.

Diese Beziehungen ergeben sich aus der Auswertung der Gleichung (3). Sie präzisieren durch Unterscheidung eines aktiven und inaktiven Teiles des Atmungssystems unsere Vorstellungen über die Faktoren, welche die Atmungsgröße bestimmen.

Die entscheidenden Parameter für den speziellen Verlauf der Temperaturfunktion sind also $\mathrm{n}$ und $\mathrm{z}$. Ihre biologische Deutung ist aber noch unklar. $z$ ist zwar definitionsgemäß die Nullpunkttemperatur, bei der also $y=0$ wird. Einer direkten Messung dürfte z nicht zugängig sein, da nicht nur in der Nähe von $z$ Stoff wechselwerte unmeßbar klein werden, sondern weil wahrscheinlich schon oberhalb von $z$ sich Abweichungen von den errechneten Werten ergeben. Da für den Nullpunkt $t=z$ ist, wird der Exponent $1 / 0$ ein Wert, der mathematisch nicht exakt faßbar ist. $z$ ist also auch nur eine rechnerische Größe. Das gleiche gilt für n. Sicher enthält n Glieder zur Anpassung an die gewählten Meßeinheiten. Bei den niederen Werten des Exponenten $1 / \mathrm{t}-\mathrm{z}$ muß $\mathrm{n}$ zwangsweise eine sehr große Zahl sein, um die Größenordnung von $\mathrm{m}$ zu erreichen.

Die Auswertung der Versuchszahlen von Јов ergab schon, daß sich $\mathrm{n}$ und $z$ im Verlaufe des Wachstums gleichsinnig, wenn auch nicht vollkommen parallel ändern.

Die Tatsache, daß zu einem niedrigen Wert von $\mathrm{z}$ auch ein geringer Wert von $\log \mathrm{n}$ gehört und umgekehrt, kommt auch beim interspezifischem Vergleich der von Krogt. untersuchten Beispiele zum Ausdruck, wie Tab. 8 zeigt.

Ein solcher Zusammenhang ist zu erwarten, da $\mathrm{n}$ und $\mathrm{z}$ antagonistisch den Temperaturquotienten bestimmen. Vergrößerung von $\mathrm{n}$ erböht den Quotien- 
Tabelle 8

Zusammenstellung der Parameterwerte für die Versuchszahlen von KrogH

\begin{tabular}{lcccc}
\hline & $\begin{array}{c}\text { Gewicht } \\
\text { in } \mathrm{g}\end{array}$ & $\log \mathrm{n}$ & $\begin{array}{c}\mathrm{z}^{\mathrm{z}} \\
\text { in }{ }^{0} \mathrm{G}\end{array}$ & $\mathrm{m}$ \\
\hline Salvelinus & 15 & 13,15 & 7,44 & 5,61 \\
Hund & - & 36,2 & 10,4 & - \\
Tenebrio & 0,163 & 71,2 & 18,9 & 142,5 \\
Carassius & 9,3 & 116,0 & 34,4 & 137,8 \\
Frosch & 35 & 101,8 & 37,2 & 191,6 \\
Kröte & 34,3 & 137,4 & 40,7 & 4262
\end{tabular}

ten, Vergrößerung von $z$ senkt ihn. Da aber nach der VAN'T Hofrschen Regel der Temperaturquotient nur in relativ schmalem Bereich schwankt, können sich beide Parameter nur in gewissen Grenzen voneinander entfernen,

Das Übergewicht hat bei der Beeinflussung des Temperaturquotienten log $\mathrm{n}$, demgegenüber ist der Einfluß einer Änderung von z geringer.

Den nicht ganz leicht zu überblickenden Zusammenhang zwischen dem temperaturbedingten Zuwachs der Atmungsgröße und $\mathrm{n}$ und $\mathrm{z}$ möchte ich aus diesem Grunde an einem speziellen Beispiel erläutern.

Unter den Versuchszahlen von KroGH fanden wir die Temperaturabhängigkeit der Atmung des Goldfisches mit den nach Gleichung (3) abgerundeten Parametern $\mathrm{z}=-34, \mathrm{~m}=142,5$ und $\log \mathrm{n}=116$. Jов gibt entsprechende Werte für einen anderen Fisch - Salvelinus - aber nicht vom gleichen Gewicht. Die zugehörigen Parameter lassen sich aber näherungsweise berechnen (Krüger 1961). $z$ ist mit $-8,5$ wesentlich niedriger. Die beiden anderen Parameter $(\mathrm{m}=4,26$ und $\log \mathrm{n}=14,89)$ kamen für unseren Zweck nicht zur Anwendung, sondern es wurde angenommen, der Goldfisch habe diesen niederen z-Wert und seine Atmungsgröße sei bei $5^{\circ} \mathrm{C}$ und $20^{\circ} \mathrm{C}$ identisch mit den Werten von KroGH. Es ist für diesen Fall dann $\log \mathrm{n}=20,7$ und $\mathrm{m}=$ 5,64 .

Die mit den angegebenen Parametern berechneten Werte finden sich in Tab. 9, außerdem ist in ihr der absolute Zuwachs für jede Temperaturstufe angegeben. Man sieht, daß dieser bei dem zugehörigen $z$-Wert von -34 mit steigender Temperatur kontinuierlich größer wird. Bei dem fiktiven $\mathrm{z}$-Wert von -8 ist dagegen der Zuwachs zwischen $5^{\circ} \mathrm{C}$ und $25^{\circ} \mathrm{C}$ nahezu linear. Im

Tabelle 9

Einfluß des Wertes von $z$ auf die beiden anderen Parameter und die Folge der berechneten Werte

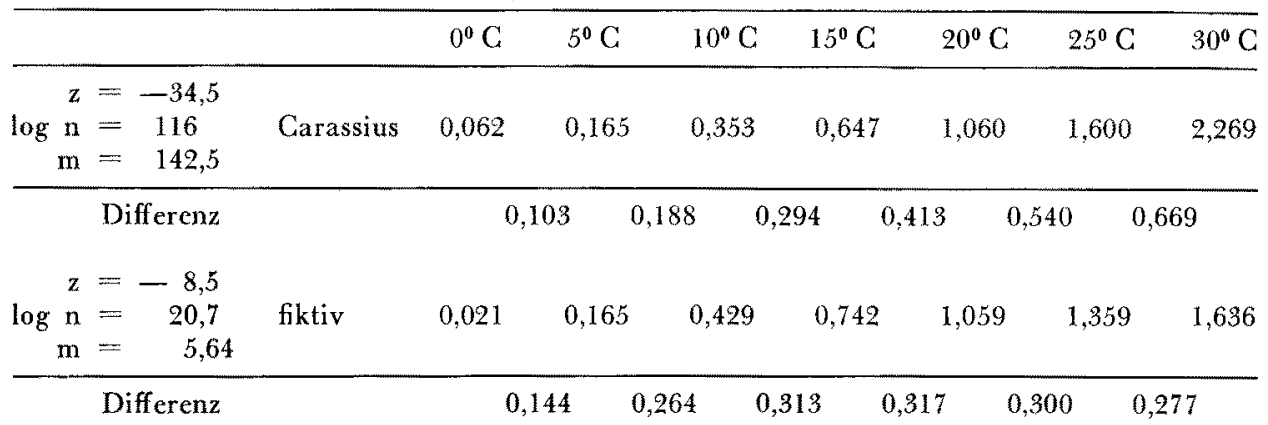

23 Meeresuntersuchungen Bd. VIII, H. 4 
ersteren Fall wird die geringe Temperaturabhängigkeit, wie sie in dem hohen $z$-Wert zum Ausdruck kommt, unterdrückt durch den hohen Wert für $\log n$. Im zweiten fiktiven Fall macht sich die starke Temperaturabhängigkeit, die der niedere $\mathrm{z}$-Wert bedingt, nur zwischen $0^{\circ} \mathrm{C}$ und $5^{\circ} \mathrm{C}$ bemerkbar. Bei den höheren Temperaturen überwiegt der Einfluß des niederen n-Wertes, der nur eine geringe Steigung zuläßt.

Von den beiden Beispielen, die hier zum Vergleich herangezogen sind, handelt es sich bei Salvelinus um einen Fisch, der vorwiegend in kühleren Gewässern lebt und für den Wassertemperaturen über $25^{\circ} \mathrm{C}$ lethal sind (FRY, Hart \& Walker 1946), während der Goldfisch auch Temperaturen von $35^{\circ} \mathrm{C}$ ertragen kann (BRETT 1946).

Die verschiedene thermische Resistenz der beiden verglichenen Fischarten findet ihren zahlenmäßigen Ausdrudk in den unterschiedlichen Parametern. Es soll an dieser Stelle noch nicht versucht werden, diese Zusammenhänge biologisch zu interpretieren. Durch den Mangel an geeignetem und zuverlässigem Zahlenmaterial dürfte eine endgültige Klärung dieses Problems der Zukunft vorbehalten sein.

Wenn es gelingt, in zahlreichen Fällen zahlenmäßig mit Gleichung (3) die Temperaturabhängigkeit biologischer Prozesse wiederzugeben, so erhebt sich die Frage, ob es sich hierbei lediglich um einen mathematischen Kunstgriff handelt, der eine Übereinstimmung zwischen Versuchszahlen und berechneten Werten herstellt. Der Versuch einer grundsätzlich abweichenden mathematischen Darstellung der Krogrsschen Normalkurve durch Jørgensen zeigt, daß dieses Ziel auf anderem Wege mit ähnlicher Genauigkeit erreicht werden kann. Mit Recht betonen Nielsen und Evans, daß es nicht möglich ist, den von ihnen eingesetzten Parametern biologische Bedeutung zuzuschreiben. In dieser Beziehung ist ohne Zweifel die Formulierung der Gleichung (3) vorzuziehen, die in Anlehnung an die Gedankengänge von Arrhenius mit ihren Parametern gewisse Vorstellungen zu verknüpfen gestattet.

In einer Formulierung, die sich lediglich darauf beschränkt, einen möglichst engen Anschluß an die Versuchswerte zu erreichen, kann man keinen wesentlichen Fortschritt sehen, wenn sie nur auf einen einzelnen oder wenige Fälle anwendbar ist. Vom mathematischen Standpunkt wäre dieses Ziel immer durch eine Reihenentwicklung mit einer genügenden Zahl Parameter zu erreichen. Die Form eines mathematischen Ausdrucks, wenn er von weiterreichender Bedeutung sein soll, muß derart sein, daß er möglichst viele Beispiele auf einheitlicher Basis auszuwerten gestattet und möglichst wenige Kenngrößen enthält. Bei Gleichung (3) scheinen diese Forderungen, soweit sich bis heute erkennen läßt, erfüllt zu sein, selbst wenn vielleicht nicht immer die gleiche Präzision der Wiedergabe der Versuchswerte erreicht wird, wie mit einer anderen Formulierung.

Herr Dr. Nicolovius machte mich vom Standpunkt des Mathematikers darauf aufmerksam, daß Gleichung (3) durch die darin enthaltenen drei Parameter sehr verschiedenartige Kurvenläufe wiederzugeben gestattet. Bei der offensichtlich sehr verschiedengestaltigen Temperaturfunktion biologischer Vorgänge muß eine geeignete Formulierung diese Eigenschaft aber von vornherein besitzen. Daß die Anpassungsfähigkeit von Gleichung (3) nicht unbegrenzt ist, zeigte die Analyse der Zahlen von Nielsen und Evans, die in ihrer Gesamtheit nicht durch Gleichung (3) dargestellt werden konnten, aber auch andere der Literatur entnommene Beispiele folgten nicht zufriedenstellend 
Gleichung (3). Hierbei bleibt aber die Frage offen, ob grundsätzlich andere Funktionen zugrunde liegen oder ob Versuchsfehler die Ursache sind.

Daß wir auch mit grundsätzlich anderen Temperaturfunktionen rechnen müssen, zeigt die mathematische Analyse der Aktivitätsatmung von Salvelinus, zu deren Darstèllung sich besser eine modifizierte Form der BERTHELOT-Gleichung eignet (KRüGER 1961).

Die drei Parameter, die Gleichung (3) enthält, sind aber die Mindestzahl, die für eine exakte mathematische Formulierung erforderlich ist, um eine eindeutige Temperaturfunktion wiederzugeben. Eine solche muß: erstens die Atmungsgröße wiedergeben, zweitens den temperaturbedingten Zuwachs und drittens die spezielle Kurvenform.

Der bislang in der Biologie angewandte $\mathrm{Q}_{10}$-Wert erfüllt von diesen Forderungen nur eine einzige - nämlich die Angabe des temperaturbedingten Zuwachses - und diese nur höchst unvollkommen. An sich wäre in allen Fällen auch die Größe a der Berthelot-Gleichung (1) anzugeben. Diese Notwendigkeit ist bislang von den Biologen übersehen worden, würde aber auch angesichts der Inkonstanz von $Q_{10}$ wenig $Z$ weck haben. Nach Gleichung (3) wird demgegenüber der temperaturbedingte Zuwachs ergänzt durch die Größe z, die die Folge der $Q_{10}$-Werte ausdrückt.

Zur Wiedergabe des temperaturbedingten Zuwachses genügen $\mathrm{n}$ und $\mathrm{z}$, denn es ist:

$$
Q_{10}=n_{n^{1 /(t+10)-z}}^{n^{1 / t-z}}
$$

m hat nur die Aufgabe, die Stoffwechselgröße quantitativ in Anpassung an Größe und Gewichtseinheit festzulegen, es dient also nur zur Fixierung der absoluten Atmungsgröße.

Die gleichen Einwände, die gegen die Anwendung des $Q_{10}$-Wertes zu machen sind, gelten auch für die ArRHENIUs-Formel.

Gleichung (3) benötigt also nur einen Parameter mehr als die bisher üblichen Darstellungsweisen, um die spezielle Kurvengestalt wiederzugeben, übertrifft sie aber in der zahlenmäßigen Darstellung bei weitem.

Trotz aller Skepsis, welche im allgemeinen Biologen mathematischer Methoden entgegenbringen, dürften die vorangegangenen Ausführungen gezeigt haben, daß es nicht ein hoffnungsloses Problem ist, geeignete Fragestellungen auch im Bereich der Biologie durch mathematische Funktionen darzustellen. Es kann aber keinem $Z$ weifel unterliegen, daß jeder Weg zu einer mathematischen Behandlung wissenschaftlicher Probleme sich als außerordentlich fruchtbar erwiesen hat. Wenn wir vom Beispiel anorganischer Wissenschaften absehen, so möchte ich an dieser Stelle für die Biologie auf die Vererbungslehre hinweisen, in der erst die Aufdeckung quantitativer Beziehungen die Fülle grundlegender Erkenntnisse ermöglichte, die wir ihr verdanken. Ohne Zweifel wird auch in der Stoffwechselphysiologie die mathematische Auswertung der Versuchsergebnisse noch zahlreiche wichtige Einsichten vermitteln.

\section{Zusammenfassung}

1. Auf die Zahlenbeispiele, die Krogh (1914) dem Entwurf seiner Normalkurve zugrunde legte, die den Zusammenhang zwischen der Geschwindig- 
keit biologischer Vorgänge und der Temperatur wiederzugeben versuchte. wird die im vergangenen Jahr vorgeschlagene Funktion:

$$
y_{t}=\frac{m}{-\frac{1}{t-z}}
$$

n

angewandt.

2. Es kann gezeigt werden, daß die neue Funktion die Zahlenbeispiele von KROGH mit hinreichender Genauigkeit wiederzugeben gestattet, so daß man in ihr den formelmäßigen Ausdruck für die Kroghsche Normalkurve sehen darf.

3. Gegenüber einem einfachen Vergleich des Kurvenverlaufes gestattet die mathematische Formel

a. einen exakten zahlenmäßigen Vergleich der gefundenen und berechneten Werte,

b. die Kennzeichnung des Kurvenverlaufes durch Zahlenwerte,

c. die Darstellung von Kurvenverläufen, die von der Normalkurve abweichen.

4. Am Beispiel von Aedes taeniorhynchus läßt sich zeigen, daß die neue Temperaturfunktion bis zu einem gewissen Umfang auch auf Entwicklungsgeschwindigkeiten anwendbar ist.

\section{Literaturverzeichnis}

1. A r rhenius, S., 1889: Über die Reaktionsgeschwindigkeit bei der Inversion von Rohrzucker durch Säuren. Z. physik. Chem., 4, 226.

2. Ba ch, G., 1958: Kritisches zur mathematischen Darstellung der Beziehung zwischen Reaktionsgeschwindigkeit und Temperatur. Pflügers Arch., 266, 447-458.

3. Berthelot, M., 1862: Essai d'une théorie sur la formation des éthers. Ann. de Chim. et de Phys., 64, Ser. III, 110-128.

4. B rett, J. R., 1946: Rate of gain of heat-tolerance in goldfish (Carassius auratus). Univ. Toronto Stud. Biol., Ser. 53.

5. Ege, R., \& $\mathrm{Krogh}, \mathrm{A} ., 1914$ : On the relation between temperature and the respiratory exchange in fishes. Intern. Rev. f. d. ges. Hydrobiol. u. Hydrogr., VII, $48-55$.

6. Fry, F. E. J., Hart, J. S., \& W a lker, K. F., 1946: Lethal temperature relations for a sample of young speckled trout, Salvelinus fontinalis. Univ. Toronto Stud. Biol., Ser. 54.

7. He int $z$ en, P., 1958: Die mathematische Behandlung der Temperaturabhängigkeit biologischer Prozesse. Eine kritische Untersudung über Berechnung und Brauchbarkeit des "Temperaturquotienten" ( $Q_{10}$-Wertes). Pflügers Arch., 266, 207-218.

8. Van't $\mathrm{H}$ of f, J. H., 1896: Studien zur chemischen Dynamik. 2. Aufl. - \& E. Cohen.

9. J a n is ch, E., 1927: Das Exponentialgesetz. Abh. z. Theorie organ. Entw., 2.

10. Job, S. V., 1955: The oxygen consumption of Salvelinus fontinalis. Univ. Toronto Biol., Ser. 61, 1-39.

11. K a n i tz, A., 1915: Temperatur und Lebensvorgänge. Berlin.

12. $\mathrm{Kr} \circ \mathrm{g} \mathrm{h}, \mathrm{A} ., 1914$ : The quantitative relation between temperature and standard metabolism in animals. Intern. $Z$. physik, chem. Biol., 1, 491-508.

13. Krüg e r, Fr, 1961: Uber den Exponenten der Temperaturfunktion biologischer Vorgänge und deren Größenabhängigkeit. Biol. Zbl. 80, 727-750.

14. Nielsen, E. T., \& Evans, D. G., 1960: Duration of the pupal stage of Aedes taeniorhynchus with a discussion of the velocity of development as a function of temperature. Oikos, 11, 200-222.

15. Precht, H., 1961: Temperaturanpassung bei wechselwarmen Tieren. Verh. d. D. Zool. Ges. Bonn, Zool. Anz. Suppl., 24, 38-60.

16. P üt te r, A., 1914: Temperaturkoeffizienten. Z. allg. Physiol, 16, 574-627. 